\title{
Evaluation of the TGS TA system for the detection of anti-cytomegalovirus antibodies
}

\author{
Annalisa Cianflone, Maria Teresa Manco, Olivia Arpino, Alessia Paganini, Massimo De Paschale, \\ Carlo Agrappi, Paola Mirri, Pierangelo Clerici
}

Microbiology Unit, Legnano Hospital, ASST Ovest Milanese, Legnano, Italy

\section{Summary}

Background and aims: The aim of the present study was to evaluate the new Technogenetics TGS TA system for detecting anti-Cytomegalovirus $\operatorname{IgG}$ and $\operatorname{IgM}$ antibodies and $\operatorname{IgG}$ avidity. The TGS TA system was compared with our routinely used system, LIAISON XL, for the detection of IgG and IgM antibodies. Only in positive IgM samples, TGS TA system was compared to an enzyme linked fluorescent assay (ELFA) test (VIDAS, BioMérieux, Marcy-l'Étoile, France) and with LIAISON XL system for the IgG avidity (if possible).

Materials and methods: Three hundred sera samples from pregnant women were examined with the TGS TA system and divided in 3 groups according to IgG and IgM screening LIAISON XL tests: 102 were non-immune women (Group 1), 98 were pregnant with past infection (Group 2) and 100 were pregnant with positive or equivocal $\operatorname{IgM}$ (95 with positive $\operatorname{IgG}$ and 5 with negative IgG) (Group 3).

Results: The overall concordance of the IgG results between LIAISON XL and TGS TA was 98.3\%: $97.1 \%$ in Group 1, 100\% in Group 2 and $98.0 \%$ in Group 3.

The overall concordance of the IgM results between LIAISON

Correspondence: Annalisa Cianflone Microbiology Unit, Legnano Hospital, ASST Ovest Milanese, via Papa Giovanni Paolo II, Legnano (MI), Italy.

Tel.: +39.0331.449319

E-mail: acianflone@libero.it

Key words: Cytomegalovirus; Pregnancy; IgM; IgG; Avidity.

Contributions: the authors contributed equally.

Conflict of interest: the authors declare no potential conflict of interest.

Received for publication: 18 January 2017.

Revision received: 15 March 2017.

Accepted for publication: 15 March 2017.

(C) Copyright A. Cianflone et al., 2017

Licensee PAGEPress, Italy

Microbiologia Medica 2017; 32:6581

doi:10.4081/mm.2017.6581

This article is distributed under the terms of the Creative Commons Attribution Noncommercial License (by-nc 4.0) which permits any noncommercial use, distribution, and reproduction in any medium, provided the original author(s) and source are credited.
XL and TGS TA was $92.1 \%$ : $100 \%$ in Group $1,99.0 \%$ in Group 2 and $70.1 \%$ in Group 3. In Group 3, the concordance between the results of the IgG avidity with the LIAISON XL and TGS TA tests was $87.4 \%$. Comparing the clinical diagnosis obtained with our protocol and that of the TGS TA system, the overall concordance was 94.3\%: 97.1\% in Group 1, 99.0\% in Group 2 and 87.0\% in Group 3.

Conclusions: In conclusion, the overall clinical concordance between the LIAISON XL/VIDAS protocol and the TGS TA system is excellent. TGA TA system shows to be a valuable tool able to clearly identify non-specific subjects, those with a non-recent infection and classify as either recent or past infection half of the subjects with undetermined infection with our protocol.

\section{Introduction}

The cytomegalovirus (CMV) is one of the leading agents responsible for congenital infections with clinical manifestations ranging from asymptomatic forms (majority of the cases) to serious damage to the foetus to, in rare cases, death by miscarriage $(5,6,12,14,20)$. Screening campaigns have been suggested in pregnant, pre-pregnant women or newborn patients for preventive purposes in addition to health education campaigns but, in general, the scientific community is divided on the opportunity of carrying out serological screening campaigns $(1,4,7,8,13,15$ 18,21,22).

However, these tests are requested in any case by many general practitioners and gynaecologists and various analytical systems are currently available for this diagnosis $(2,9-11,19)$ such as enzyme-linked immunosorbent assay (ELISA), chemiluminescence immunoassay (CLIA) and enzyme linked fluorescent assay (ELFA) which are able, depending on the instrument, to process a great number of samples in a short time. The commercial tests available generally have excellent sensitivity and specificity levels, with different formulations and the possible use of distinct antigens (natural, recombinant or synthetic one) which may be responsible for slight differences in the results obtained when comparing the available tests. The extent of these differences must be known in advance.

In this study we compared the CMV serology results obtained with our standard internal laboratory protocol, to the CMV results from the TGS TA system. In accordance with our laboratory protocol, on all samples with a positive or equivocal CMV IgM result obtained with LIAISON XL (DiaSorin, Saluggia, Italy), CMV $\operatorname{IgM}$ is also determined in a second round with VIDAS (BioMérieux, Marcy-l'Étoile, France) and a CMV IgG avidity analysis is performed on LIAISON XL. 


\section{Materials and Methods}

Three hundred sera samples from women who came to our centre for the normal follow-up in pregnancy were examined and divided into the groups below:

Group 1: 102 samples from non-immune pregnant women

Group 2: 98 samples from pregnant women with past infection

Group 3: 100 samples from pregnant women with positive or equivocal IgM (95 with positive $\operatorname{IgG}$ and 5 with negative $\operatorname{IgG}$ ).

Routinely, samples are tested using chemiluminescence LIAISON XL system for IgG and IgM (LIAISON CMV II IgG and CMV II IgM; DiaSorin, Italy). In positive subjects for IgM, samples are tested using an ELFA test (VIDAS Toxo IgM; BioMérieux, France). The avidity of the IgG (if possible) is determined with LIAISON XL (LIAISON CMV Avidity II; DiaSorin, Italy)

All samples are tested with TGS TA system for detecting IgG and IgM and, for Group 3, IgG avidity (TGS TA CMV IgG, TGS TA CMV IgM, TGS TA CMV IgG Avidity; Technogenetics, Milan, Italy).

For Groups 1 and 2, the TGS TA system was used on fresh samples immediately after the results with LIAISON XL test while, for Group 3, TGS TA was used on frozen samples stored on the basis of the results with our protocol.

The TGS TA CMV IgG and TGS TA CMV IgM kit employs an indirect two-step immunological method based on the principle of chemiluminescence. The solid phase is coated with a specific antigen and the conjugate is or an anti-human IgG antibody or antihuman $\operatorname{IgG}$ antibody labelled with an acridinium ester derivative. The TGS TA CMV IgG avidity uses a borate buffer able to prevalently break the binding Antigen ( $\mathrm{Ag}$ )- Antibody (Ab) if the antibody is at low avidity.

The Interpretation scheme for the different tests are shown in Tables 1 and 2 .

With reference to the $\operatorname{IgG}$ avidity, low avidity is strongly indicative of an infection occurring in the past 3 months for both systems; although high avidity does not exclude the possibility of a recent infection, it is strongly indicative of an infection at least 3 months before for both methods. The interpretation for moderate avidity is doubtful and the infection cannot be dated.

\section{Results}

The overall concordance and concordance in the three groups resulting from a comparison of the results obtained with the TGS TA and LIAISON XL systems is shown in Table 3.

Fifty-two samples were negative to the TGS IgM test in Group 3 (samples with positive or equivocal IgM for the LIAISON XL system). These samples were examined with the VIDAS IgM test and 23 were negative, 15 positive and 14 equivocal (Table 4).

In addition, the avidity was determined with both LIAISON XL and TGS TA in the 95 samples of Group 3 with IgG and the concordance was $87.4 \%$ (Table 5).

As far as the clinical assessment is concerned, the TGS TA system found 3 samples (2.9\%) with low IgG titre (values 11.4-18.8 $\mathrm{UA} / \mathrm{mL}$ ) in Group 1 (non-immune women). All these subjects had values between 5.5 and $11.8 \mathrm{AU} / \mathrm{mL}$ for the LIAISON XL system. The clinical concordance is $97.1 \%$.

The TGS TA test detected one case with positive IgM (not confirmed with VIDAS IgM) and low avidity with TGS TA and moderate with LIAISON XL in Group 2 (immune women). The clinical concordance is $99.0 \%$.

Based on our protocol, patients in Group 3 (IgM positive or equivocal samples for LIAISON XL IgM) were classified as: 16 patients with a recent infection (samples confirmed with the VIDAS IgM and low avidity with LIAISON XL), of whom 3 without IgG; 14 with a non-determinable infection as they had moderate avidity with LIAISON XL (samples confirmed with the VIDAS IgM); 43 patients with past infection (samples confirmed with the VIDAS IgM and high avidity with LIAISON XL); 25 patients with IgM supposed non-specific not confirmed with the VIDAS IgM and high avidity with LIAISON XL; 2 non-immune patients, without IgG and IgM not confirmed with VIDAS IgM.

The interpretations obtained with the TGS TA system (IgG, $\operatorname{IgM}$ and $\operatorname{IgG}$ avidity) in these sub-groups are shown in Table 6. The concordance is $87.0 \%$.

Considering all three groups, the clinical concordance is shown in Table 7.

Table 1. Interpretation scheme for IgG and IgM anti-cytomegalovirus with LIAISON XL systems, VIDAS and TGS TA.

\begin{tabular}{lccccc}
$\begin{array}{l}\text { Interpretation } \\
\text { scheme }\end{array}$ & $\begin{array}{c}\text { IIAISON XI, } \\
\text { IU/mL }\end{array}$ & $\begin{array}{c}\text { TGS TA, } \\
\text { AU/mL }\end{array}$ & $\begin{array}{c}\text { LIAISON XI, } \\
\text { AU/mL }\end{array}$ & $\begin{array}{c}\text { IgM } \\
\text { VIDAS } \\
\text { (index) }\end{array}$ & $\begin{array}{c}\text { TGS TA, } \\
\text { AU/mL }\end{array}$ \\
Negative & $<12$ & $<10$ & $<18$ & $<0.70$ & $<6$ \\
Equivocal & $12-14$ & - & $18-22$ & $0.70-0.90$ & - \\
\hline Positive & $>14$ & $\geq 10$ & $>22$ & $>0.90$ & $\geq 6$ \\
\hline
\end{tabular}

Table 2. Interpretation scheme for the anti-cytomegalovirus IgG avidity tests with the LIAISON XL and TGS TA.

\begin{tabular}{lcc} 
Interpretation scheme & $\operatorname{Ig} G$ avidity & TGS TA (index) \\
Low & LIAISON XI (index) & $<0.150$ \\
Moderate & $<0.150$ & $0.150-0.200$ \\
\hline High & $0.150-0.250$ & $\geq 0.200$ \\
\hline
\end{tabular}




\section{Discussion and Conclusions}

The concordance between LIAISON XL and TGS TA in the IgG tests is excellent in the three groups. The few discrepancies are due to the $\operatorname{IgG}$ values close the cut-off. The concordance for the IgM tests is excellent in the first two groups; in the third group, the discordance is greater, also because there were subjects with nonspecific IgM in this group. Subjects with IgM positive in LIAISON XL (also with VIDAS) and negative in the TGS TA test, the avidity, in all cases except one, was high with both LIAISON XL and TGS TA. It should be recalled that the TGS TA system for IgM does not have a grey doubtful area around the cut-off, unlike the
LIAISON XL and VIDAS, therefore the calculation of the concordance suffers from this limitation.

The concordance between LIAISON XL and TGS TA in the IgG avidity tests is good. Most of samples with moderate avidity with LIAISON XL test, shown a high or low avidity values with TGS TA system. Therefore, the indeterminate infections found with our protocol could be defined as recent or past with TGS TA method.

A limit of the study design is the lack of cases of recent CMV infection due to low incidence of acute infection in pregnant women in our area (3) that makes quite rare the observation of recent infection rare. Another limitation of this study is the selection of samples in Group 3 using our protocol which provides screening for IgG and IgM with LIAISON XL. On the other hand,

Table 3. Overall concordance and concordance in the three groups between LIAISON XL and TGS TA for the detection of anticytomegalovirus IgG and IgM in samples from pregnant women.

\begin{tabular}{|c|c|c|c|c|}
\hline & & & & \\
\hline & TGS TA neg & TGS TA pos & TGS TA neg & TGS TA pos \\
\hline Overall concor & nples from pr & & & \\
\hline LIAISON XL neg & 102 & 5 & 199 & 1 \\
\hline LIAISON XL pos & 0 & 193 & 20 & 47 \\
\hline LIAISON XL eq & - & - & 32 & 1 \\
\hline Group 1: conco & imples from I & regnant wom & & \\
\hline LIAISON XL neg & 99 & 3 & 102 & 0 \\
\hline LIAISON XL pos & 0 & 0 & 0 & 0 \\
\hline
\end{tabular}

\begin{tabular}{lcccc} 
LIAISON XL neg & 0 & 0 & 97 & 1 \\
LIAISON XL pos & 0 & 98 & 0 & 0 \\
\hline Group 3: concordance in $\mathbf{1 0 0}$ samples from pregnant women with positive or equivocal IgM with LIAISON XL ****
\end{tabular}

\begin{tabular}{lcccc} 
LIAISON XL neg & 3 & 2 & 0 & 0 \\
LIAISON XL pos & 0 & 95 & 20 & 47 \\
\hline LIAISON XL eq & - & - & 32 & 1 \\
\hline
\end{tabular}

*Overall concordance (excluding equivocal cases): IgG 98.3\%; IgM 92.1\%. ** Overall concordance: IgG 97.1\%; IgM 100\%. *** Overall concordance: IgG 100\%; IgM 99\%. **** Overall concordance (excluding equivocal cases): $\operatorname{IgG} 98 \% ; \operatorname{IgM} 70.1 \%$.

Table 4. Results of the VIDAS on 52 samples equivocal or positive to the LIAISON XL anti-cytomegalovirus IgM and negative with the TGS TA IgM.

\begin{tabular}{lccc}
\hline IgM positive or equivocal for the LIAISON XIL system & VIDAS & TGS TA \\
Negative & 0 & 23 & 52 \\
Positive & 20 & 15 & 0 \\
\hline Equivocal & 32 & 14 & 0
\end{tabular}

Table 5. Concordance between the LIAISON XL and TGS TA anti-cytomegalovirus IgG avidity tests in 95 individuals positive or equivocal (with IgG positive) for LIAISON XL IgM.

\begin{tabular}{lcccc} 
Avidity & Low & TGSTGS TA avidity & Hoderate & \\
& & & \\
LIAISON XL & & & 1 & 13 \\
Low & 11 & 1 & 4 & 14 \\
Moderate & 5 & 5 & 67 & 68 \\
High & 0 & 1 & 72 & 95 \\
Total & 16 & 7 & & \\
\hline
\end{tabular}

Concordance: $87.4 \%$. 
Table 6. Comparison between the clinical interpretations given with cytomegalovirus LIAISON XL/VIDAS and TGS TA systems.

\begin{tabular}{lccccc}
\hline LIAISON XI/VIDAS system & $\begin{array}{c}\text { Probable recent } \\
\text { infection }\end{array}$ & $\begin{array}{c}\text { Undeterminate } \\
\text { infection }\end{array}$ & $\begin{array}{c}\text { TGS TA system } \\
\text { Probable } \\
\text { non-recent infection }\end{array}$ & Non immune \\
Probable recent infection & 13 & 1 & 1 & 1 & 16 \\
Undeterminate infection & 5 & 5 & 4 & 0 & 0 \\
\hline Probable non-recent infection & 0 & 1 & 67 & 2 & 14 \\
Non immune & 0 & 0 & 72 & 3 & 2 \\
\hline Total & 18 & 7 & 0 & 100 \\
\hline
\end{tabular}

Concordance $87.0 \%$.

Table 7. Overall clinical agreement between the results obtained with the cytomegalovirus LIAISON XL/VIDAS protocol and those of the TGS TA system.

\begin{tabular}{lccccc} 
LIAISON XI/ & $\begin{array}{c}\text { Probable recent } \\
\text { infection }\end{array}$ & $\begin{array}{c}\text { Undeterminate } \\
\text { infection }\end{array}$ & $\begin{array}{c}\text { TGS TA system } \\
\text { Probable } \\
\text { non-recent infection }\end{array}$ & Non immune \\
\hline Probable recent infection & 13 & 1 & 1 & 1 & 16 \\
Undeterminate infection & 6 & 5 & 4 & 0 & 15 \\
\hline Probable non-recent infection & 0 & 1 & 164 & 0 & 101 \\
Non immune & 0 & 0 & 3 & 102 & 104 \\
\hline TOTAL & 19 & 7 & 172 & 300 \\
\hline
\end{tabular}

Total concordance $94.3 \%$.

our protocol leads to the definition of the type of infection that was compared with the interpretation given by the TGS TA system. The clinical interpretation correlates well between our protocol and TGS TA system. Taking into account all three groups, the TGS TA system clearly identifies many subjects non-specific and those with non-recent infection. Further, although it missed two cases of recent infection in Group 3, it found one more in Group 2 compared to our protocol. In addition, more than half of samples with undetermined infection with our protocol are clearly defined by TGS TA as either recent or past infection.

In conclusion the overall clinical concordance between the two systems is good and the TGS TA is a useful system to evaluate the immune status related to CMV infection.

\section{References}

1. Ahlfors K, Ivarsson SA, Harris S. Report on a long-term study of maternal and congenital cytomegalovirus infection in Sweden. Review of prospective studies available in the literature. Scand J Infect Dis 1999;31:443-57.

2. De Paschale M, Agrappi C, Manco MT, Clerici P. Positive predictive value of anti-HCMV IgM as an index of primary infection. J Virol Methods 2010;168:121-5.

3. De Paschale M, Agrappi C, Manco MT, et al. Incidence and risk of cytomegalovirus infection during pregnancy in an urban area of Northern Italy. Infect Dis Obstet Gynecol 2009;206505.

4. Enders G, Bäder U, Lindemann L, et al. Prenatal diagnosis of congenital cytomegalovirus infection in 189 pregnancies with known outcome. Prenat Diagn 2001;21:362-77.

5. Fowler KB, Stagno S, Pass RF, et al. The outcome of congenital cytomegalovirus infection in relation to maternal antibody status. N Engl J Med 1992;326:663-7.
6. Fowler KB, Stagno S, Pass RF. Maternal immunity and prevention of congenital cytomegalovirus infection. JAMA 2003;289:1008-11.

7. Gratacap-Cavallier B, Morand P, Dutertre N, et al. Cytomegalovirus infection in pregnant women. Seroepidemiological prospective study in 1,018 women in Isere. J Gynecol Obstet Biol Reprod (Paris) 1998;27:161-6.

8. Hagay ZJ, Biran G, Ornoy A, Reece EA. Congenital cytomegalovirus infection: a long-standing problem still seeking a solution. Am J Obstet Gynecol 1996;174:241-5.

9. Kaneko M, Ohhashi M, Minematsu T, et al. Maternal immunoglobulin $\mathrm{G}$ avidity as a diagnostic tool to identify pregnant women at risk of congenital cytomegalovirus infection. Infect Chemother 2017;23:173-6.

10. Lazzarotto T, Gabrielli L, Lanari M, et al. Congenital cytomegalovirus infection: recent advances in the diagnosis of maternal infection. Hum Immunol 2004;65:410-5.

11. Lazzarotto T, Guerra B, Gabrielli L, et al. Update on the prevention, diagnosis and management of cytomegalovirus infection during pregnancy. Clin Microbiol Infect 2011;17:1285-93.

12. Leruez-Ville M, Ville Y. Fetal cytomegalovirus infection. Best Pract Res Clin Obstet Gynaecol 2017;38:97-107.

13. Mazeron MC. Conséquence néonatales des maladies sexuellement trasmises. Conduite à tenir devant une infection à cytomegalovirus. Med Mal Infect 1994;24:485-95.

14. Mestas E. Congenital cytomegalovirus. Adv Neonatal Care 2016;16:60-5.

15. Peckham CS, Chin KS, Coleman JC, et al. Cytomegalovirus infection in pregnancy: preliminary findings from a prospective study. Lancet 1983;i:1352-3.

16. Revello MG, Gerna G. Diagnosis and management of human cytomagalovirus infection in the mother, fetus, and newborn infant. Clin Microbiol Rev 2002;15:680-715.

17. Revello MG, Tibaldi C, Masuelli G, et al. Prevention of pri- 
mary cytomegalovirus infection in pregnancy. EBioMedicine 2015;2:1205-10.

18. Schoub BD, Johnson S, McAnerney JM, et al. Is antenatal screening for rubella and cytomegalovirus justified? S Afr Med J 1993;83:108-10.

19. Sellier Y, Guilleminot T, Ville Y, et al. Comparison of the LIAISON(®) CMV IgG Avidity II and the VIDAS(®) CMVIgG Avidity II assays for the diagnosis of primary infection in pregnant women. J Clin Virol 2015;72:46-8.
20. Stagno S, Pass RF, Cloud G, et al. Primary cytomegalovirus infection in pregnancy : Incidence, transmission to fetus and clinical outcome. JAMA 1986;256:1904-8.

21. Thackeray R, Magnusson BM. Women's attitudes toward practicing cytomegalovirus prevention behaviors. Prev Med Rep 2016;4:517-24.

22. Yow MD, Demmler GJ. Congenital cytomegalovirus disease 20 years is long enough. N Engl J Med 1992;326:702-3. 